Case Report

\title{
A Rare Clinical Case: Giant Splenic Artery Aneurysm and Its Successful Endovascular Treatment
}

\author{
Alptekin Yasim, ${ }^{1}$ Hakan Kara, ${ }^{2}$ and Erdinc Eroglu ${ }^{1}$ \\ ${ }^{1}$ Faculty of Medicine, Kahramanmaras Sutcu Imam University, Kahramanmaras, Turkey \\ ${ }^{2}$ Giresun Ada Hospital, Giresun, Turkey \\ Correspondence should be addressed to Alptekin Yasim; alpyasim@hotmail.com
}

Received 25 January 2017; Accepted 13 June 2017; Published 13 July 2017

Academic Editor: Nikolaos Papanas

Copyright (C) 2017 Alptekin Yasim et al. This is an open access article distributed under the Creative Commons Attribution License, which permits unrestricted use, distribution, and reproduction in any medium, provided the original work is properly cited.

Objectives. The true giant splenic artery aneurysms are extremely rare and can be fatal. Although surgical approach has been the standard of care in the past, endovascular methods gained more importance in recent years. Background. We describe a case with true giant splenic artery aneurysm, treated with endovascular approach. Methods. A 68-year-old female patient with abdominal pain admitted to our clinic had true splenic artery aneurysm $(14 \times 10 \times 9 \mathrm{~cm})$. We decided on endovascular treatment using vascular plug and the treatment was performed successfully. Conclusions. Due to high mortality and morbidity in open surgery, endovascular treatment of giant splenic artery aneurysm is a better treatment option.

\section{Introduction}

The splenic artery aneurysm (SAA) is the third most common intraabdominal aneurysm following abdominal aorta aneurysms and iliac artery aneurysm. It constitutes more than $60 \%$ of the all visceral artery aneurysms [1-4]. It is reported $0.8-1 \%$ in prevalence arteriography and $0.001-0.2 \%$ in autopsy series $[1,5,6]$. Although most cases are asymptomatic, risk of rupture increases in aneurysms larger than $2 \mathrm{~cm}$. The risk of rupture ranges from $2 \%$ to $10 \%[1,5-7]$ and risk increases in presence of portal hypertension, in liver transplant patients, and during pregnancy $[1,5,8,9]$. In addition, the risk of rupture is high in men and in smokers [7]. Occasionally aneurysm can erode on adjacent organs or into pancreatic duct and present as gastrointestinal bleeding [2]. Rupture is the catastrophic complication of SAAs and causes $10-100 \%$ mortality $[1,4,5,7]$.

In order to prevent risk of rupture, ACC/AHA guidelines recommended that treatment is indicated in asymptomatic aneurysm that is larger than $2 \mathrm{~cm}$ [7]. Additionally, treatment is indicated in patients with symptomatic aneurysm, pregnancy and portal hypertension and woman in childbearing age even if the aneurysm is smaller than $2 \mathrm{~cm}[1,8]$.

SAAs are generally smaller than $3 \mathrm{~cm}$. If aneurysm is greater than $10 \mathrm{~cm}$, they are called giant SAA. These aneurysms can be true or pseudoaneurysms. But aneurysms greater than $10 \mathrm{~cm}$ are rarely seen.

Surgery (open or laparoscopic) has been used as treatment method for many years. But recently endovascular treatments (stent-graft implantation, coil embolization, plug deployment, or glue embolization) are used as these treatment methods are often more successful. The purpose of this study is to present a case with true giant splenic artery aneurysm that had endovascular treatment.

\section{Case Report}

A 68-year-old woman patient was referred to our clinic following detection of abdominal aneurysm after USG examination. She suffered from abdominal discomfort. She was congenitally deaf and dumb. Other physical findings were normal. Her past medical history revealed occasional abdominal pain and constipation for the past 6 months. There was no risk factor such as hypertension, diabetes, smoking, or atherosclerosis. Patient was not using any medical treatment and had no history of trauma or surgery. There was no history of any systemic infections, family history of aneurysm, and connective tissue disorders. She was nullipara.

All laboratory tests were within the normal range. 


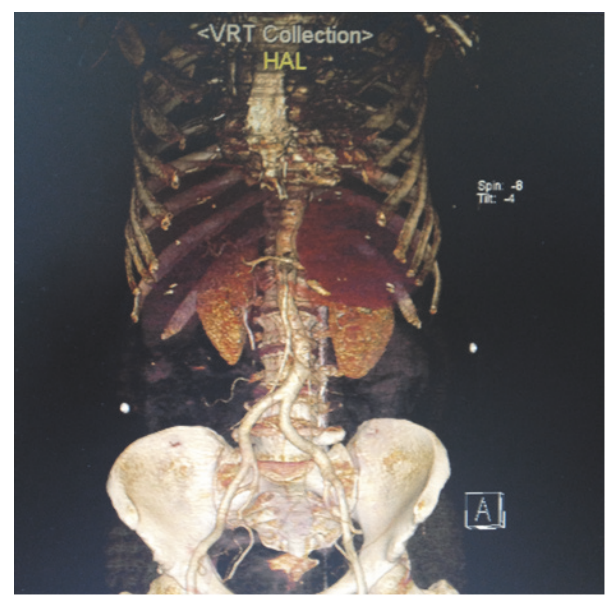

FIGURE 1: Computed tomographic angiography revealed aneurysmatic expansion.

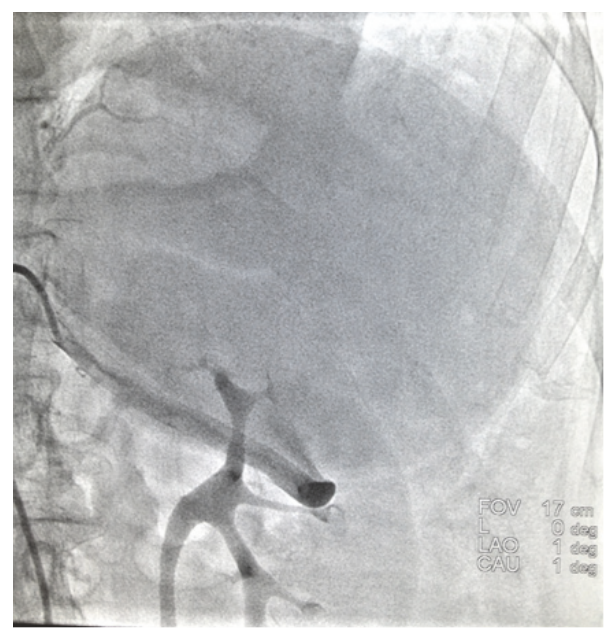

FIgURE 2: Angiogram showed giant splenic artery aneurysm.

A computed tomographic angiography revealed $14 \times$ $10 \times 9 \mathrm{~cm}$ sized regular bounded aneurysmatic expansion placed between pancreas tail, spleen, stomach, and kidney in left upper quadrant of abdomen. It causes compression on pancreas tale, probably splenic artery sourced and in close connection with distal part of splenic artery bifurcation (Figure 1).

A selective angiogram was performed and showed splenic artery expanding to $6.5 \mathrm{~mm}$ diameter and confirmed the presence of true giant saccular splenic aneurysm arising from splenic artery and expanding to the superior (Figure 2), after aneurysm splenic artery became rudimentary.

The patient was scheduled for endovascular intervention. Stent-graft implantation was not possible due to artery diameter being too wide at proximal portion and small in distal portion. Coil or glue embolization also was not possible due to wide aneurysm. Eventually, to close the feeding artery with vascular plug is decided. A right common femoral artery approach was performed under local anesthesia; a 7French sheath was placed. A 7-French guiding catheter was

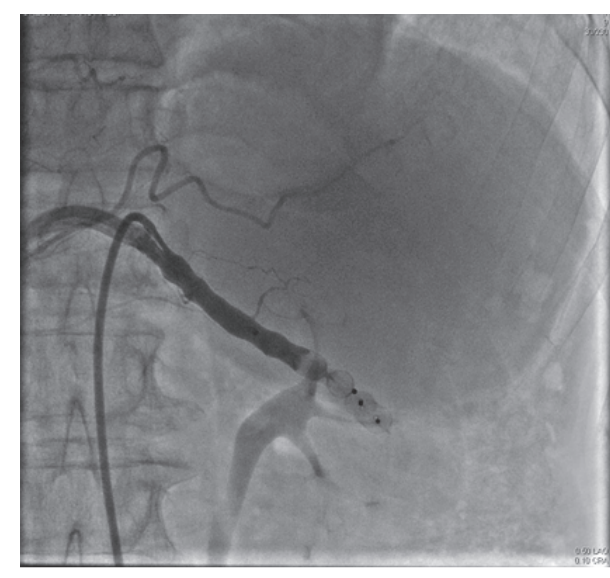

FIGURE 3: Angiogram after vascular plug employment.

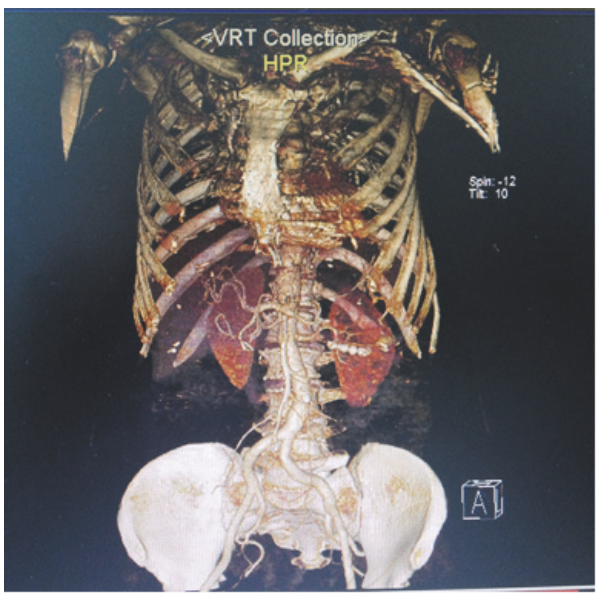

FIGURE 4: After three months, CT angiography showed thrombosed aneurysm sack and normal spleen.

positioned at the origin of the splenic artery over $0.035^{\prime \prime} \times$ $260 \mathrm{~cm}$ guidewire. Splenic angiogram was obtained again and reference values were recorded by contrast injection. After $7 \mathrm{~mm}$ balloon expanded inside of the splenic artery over $0.014^{\prime \prime}$ inch floppy wire and long injection performed to celiac trunk. No retrograde flow was observed inside of the aneurysm. $8 \mathrm{~mm}$ Amplatzer vascular plug (St. Jude Medical, Austin, TX) was deployed in aneurysm entry. Minimal leakage was observed after contrast injection. A $10 \mathrm{~mm}$ Amplatzer vascular plug was deployed proximally of the $8 \mathrm{~mm}$ plug. No retrograde filling in to the aneurysm and no leakage were observed in contrast injection from celiac trunk (Figure 3).

Patient was discharged at second day after the procedure. At the third month, CT angiography showed thrombosed aneurysm sack and no problem with spleen (Figure 4). Patient was clinically comfort with no complaint.

\section{Discussion}

SAAs are the most common visceral artery aneurysms and their etiology is unclear. Pregnancy, portal hypertension, 
cirrhosis, liver transplantation, hypertension, atherosclerosis, medial fibrodisplasia, splenomegaly, pancreatic pseudocyst, and vascular collagen diseases are the risk factors for SAA development $[1,2,4,8]$.

Although it is more common in women 4 times greater than men, rupture is more common in men [4]. Regarding the developments and more common usage of medical imaging technologies, we encounter small SAAs more often and treat these small SAAs successfully with endovascular methods. SAAs are generally smaller than $3 \mathrm{~cm}$ and they are called giant SAAs if aneurysm is greater than $10 \mathrm{~cm}[2,3]$. Giant SAAs are extremely rare cases. Only 12 giant SAAs were reported in the literature search in 2005 [3]. However, it is not reported that these aneurysms are true or pseudoaneurysms. Recently, in another study, 10 true giant SAAs were reported [6].

These giant aneurysms were treated by surgery in the past years $[2,3]$ due to lack of endovascular techniques. It is nearly impossible to treat giant SAAs with stent grafts due to place of occurrence of SAA that is generally located at distal portion of splenic artery, because the diameter difference before and after aneurysm is great and there is no enough place on the distal to aneurysm to implant stent graft unlike in our case.

In this case due to large diameter of aneurysm sack and high flow, coil embolization was not an option. Guziński et al. treated a giant aneurysm with glue embolization into splenic artery after cutting off the blood flow [6]. We planned to cut off arterial flow by vascular plug. The important point here was whether or not spleen will go to autosplenectomy. However, splenectomy was performed in most of the surgical procedures in the past. Although we were going to cut off blood flow in spleen without splenectomy, there was a risk of splenic infarct. However spleen has rich collateral and short gastric artery feeding; it is reported that this may develop splenic infarct and postembolization syndrome $[5,9,10]$. But it is mentioned that after this procedure splenic insufficiency is rare [4]. Li et al. performed aneurysm repair to 35 patients with SAA by preserving splenic artery in some of them, whereas they occluded splenic artery in the rest of the patients in their study [5]. Postembolization syndrome consists of abdominal pain, fever, and vomiting symptoms occurring in $15 \%$ of the patients where they preserved splenic artery and $37 \%$ of the patients where they did not. However, they reported no splenic artery insufficiency in both groups and it is safe to perform embolization in splenic artery. We occluded splenic artery from the proximal portion of the aneurysm in the light of these findings. We did not observe postembolization syndrome with our patient.

\section{Conclusion}

This case report is unique endovascular treatment by vascular plug of giant SAA in the literature. Splenic artery can be occluded safely in these particular cases. By these ways, patients exposure to surgical morbidity and mortality can reduce.

\section{Disclosure}

This study was presented at the 17th Annual Congress on Turkish Vascular and Endovascular Surgery, Antalya, Turkey, October 29-November 1, 2015.

\section{Conflicts of Interest}

The authors declare that they have no conflicts of interest.

\section{References}

[1] A. Abdulrahman, A. Shabkah, M. Hassanain, and M. Aljiffry, "Ruptured spontaneous splenic artery aneurysm: A case report and review of the literature," International Journal of Surgery Case Reports, vol. 5, no. 10, pp. 754-757, 2014.

[2] S. Ali, V. Verma, S. R, and I. Wani, "Giant Splenic Artery Aneurysm: Case Report," ISRN Surgery, vol. 2011, Article ID 383450, 3 pages, 2011.

[3] R. Pescarus, B. Montreuil, and Y. Bendavid, "Giant splenic artery aneurysms: Case report and review of the literature," Journal of Vascular Surgery, vol. 42, no. 2, pp. 344-347, 2005.

[4] A. M. Ierardi, M. Petrillo, A. Bacuzzi et al., "Endovascular retreatment of a splenic artery aneurysm refilled by collateral branches of the left gastric artery: A case report," Journal of Medical Case Reports, vol. 8, no. 1, article no. 436, 2014.

[5] E.-S. Li, J.-X. Mu, S.-M. Ji et al., “Total splenic artery embolization for splenic artery aneurysms in patients with normal spleen," World Journal of Gastroenterology, vol. 20, no. 2, pp. 555-560, 2014.

[6] M. Guziński, J. Kurcz, M. Kukulska, M. Neska, and J. Garcarek, "Embolization of a true giant splenic artery aneurysm using NBCA glue - Case report and literature review," Polish Journal of Radiology, vol. 80, no. 1, pp. 155-158, 2015.

[7] K. Marmagkiolis, F. Gobal, D. Mego, M. Cilingiroglu, and W. Rollefson, "Endovascular management of splenic arterial aneurysms," International Journal of Cardiology, vol. 174, no. 1, pp. 146-149, 2014.

[8] A. Kuklinski, K. Btycki, W. Matuszewski, A. Ostrach, Z. Kupis, and T. Legowik, "Embolisation of a large, symptomatic splenic artery pseudoaneuysm," Polish Journal of Radiology, vol. 79, pp. 194-198, 2014.

[9] E. A. Rogriguez-Caulo, O. Araji, N. Miranda, J. C. Tellez, and C. Velazquez, "Fusiform giant splenic artery aneurysm," Cirugia Espanola, vol. 92, pp. 209-218, 2014.

[10] R. Loffroy, B. Guiu, J.-P. Cercueil et al., "Transcatheter Arterial Embolization of Splenic Artery Aneurysms and Pseudoaneurysms: Short- and Long-Term Results," Annals of Vascular Surgery, vol. 22, no. 5, pp. 618-626, 2008. 


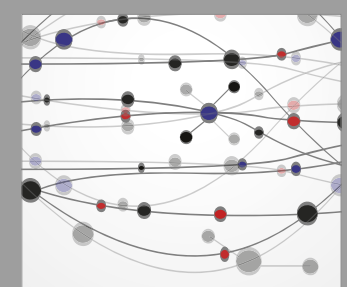

The Scientific World Journal
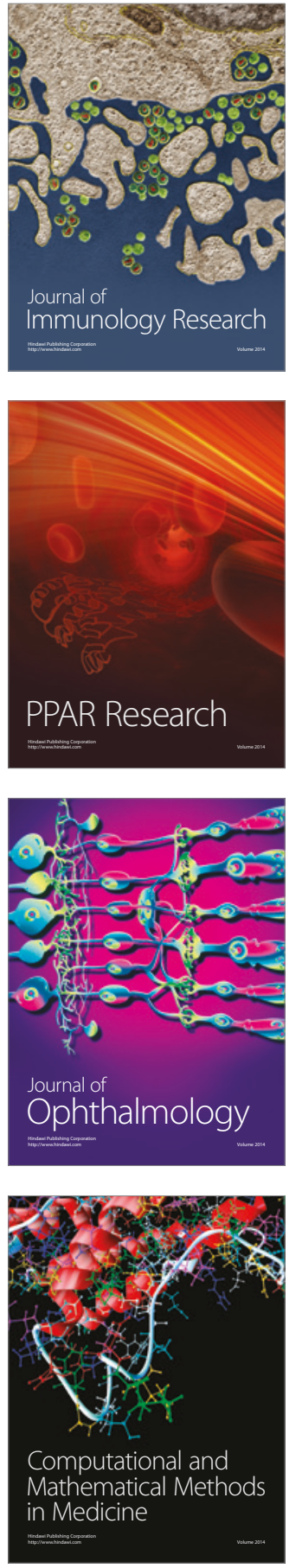

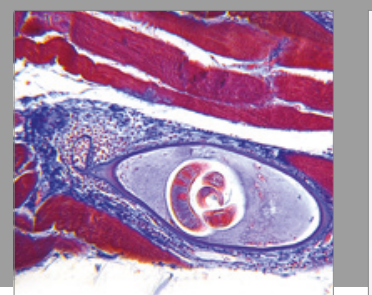

Gastroenterology Research and Practice
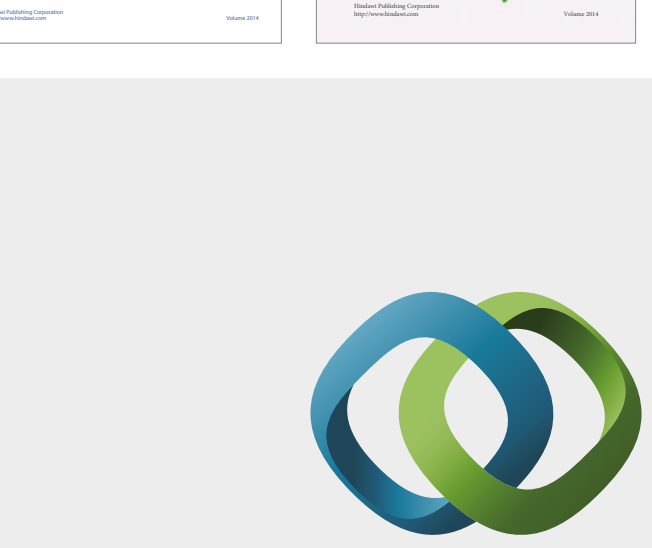

\section{Hindawi}

Submit your manuscripts at

https://www.hindawi.com
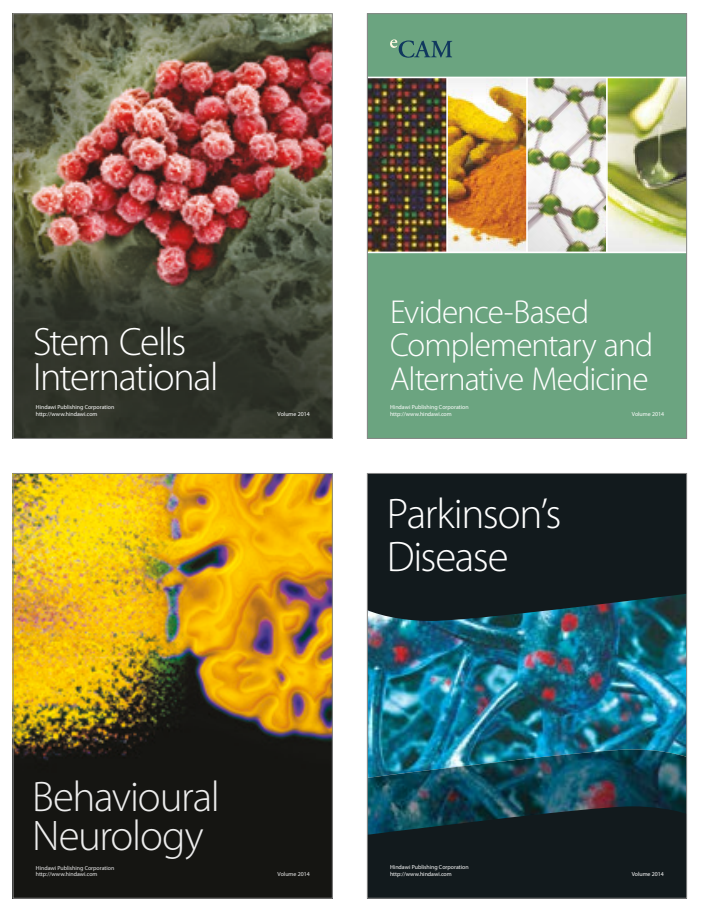
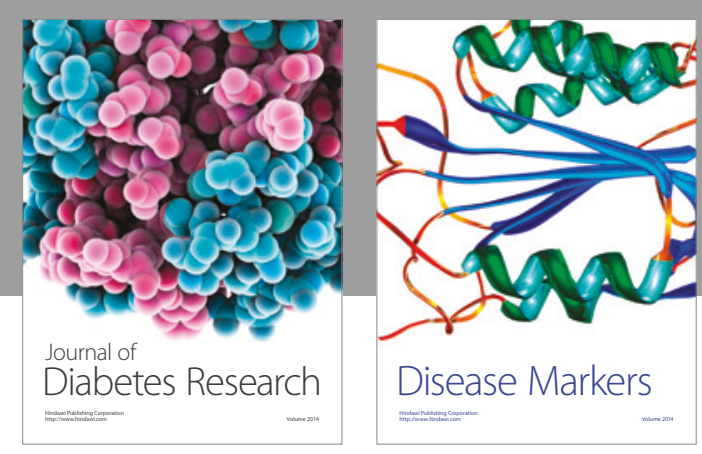

Disease Markers
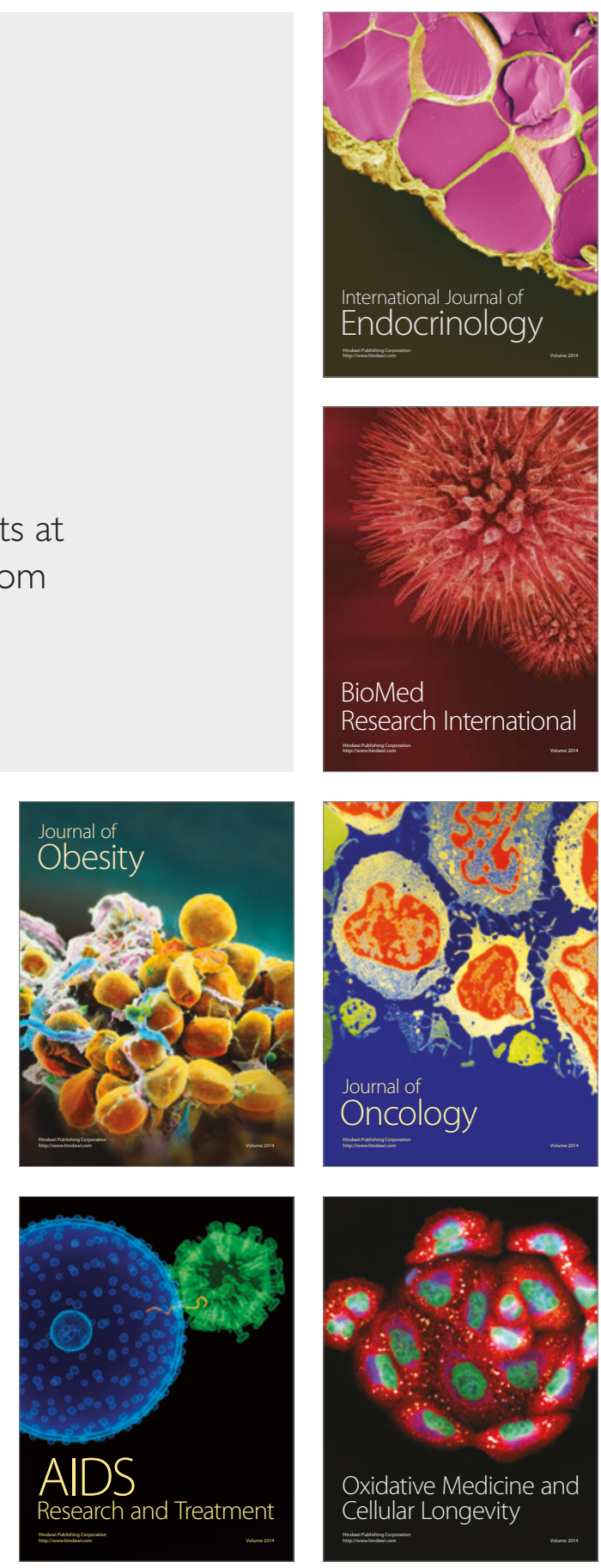